\title{
miR-139-5p functions as a tumor suppressor in cervical cancer by targeting TCF4 and inhibiting $\mathrm{Wnt} / \beta$-catenin signaling
}

This article was published in the following Dove Press journal: OncoTargets and Therapy

Xia Ji ${ }^{1}$

Hairong Guo'

Shanyu Yin ${ }^{2}$

Haiyan Du'

'Department of Obstetrics and Gynecology, Inner Mongolia Baogang Hospital, Baotou, Inner Mongolia 014010 , People's Republic of China; ${ }^{2}$ Department of Ultrasonography, The First Affiliated Hospital of Zhejiang University, Hangzhou 310003, People's Republic of China
Correspondence: Haiyan Du Department of Obstetrics and Gynecology, Inner Mongolia Baogang Hospital, 20 Shaoxian Road Kundulun District, Baotou City, Inner Mongolia 0I40 I0, People's Republic of China Email yanzil959II@I63.com
Objectives: Dysregulation of microRNAs (miRNAs) has been recognized as a crucial biological event in the development of cervical cancer (CC). miR-139-5p was identified as a significant tumor suppressor in multiple human cancers, leaving its roles and mechanisms in $\mathrm{CC}$ absolutely unclear. We aimed to investigate the implication of miR-139-5p in CC progression.

Methods: miR-139-5p expression in 40 paired CC tissues and several cell lines was determined by qRT-PCR firstly. The implications of miR-139-5p in CC cell proliferation and migration were revealed by CCK-8, EdU and transwell assays, respectively. The mechanism underlying the tumor-suppressing roles of miR-139-5p in CC was investigated sequentially by dual luciferase, qRT-PCR, and Western blot analysis. The expression of transcription factor 4 (TCF4), the validated target of miR-139-5p from our experiments, was finally detected by qRT-PCR and immunohistochemistry in CC tissues, and its expression correlates with miR-139-5p was explored.

Results: We found that miR-139-5p expression was frequently decreased in CC tissues and cell lines, and its lower level was associated with positive lymph node metastasis. Cellular assays proved the significant tumor-suppressing roles of miR-139-5p by inhibiting CC cell proliferation and migration, and markedly blocking $\mathrm{Wnt} / \beta$-catenin signaling. Since bioinformatics analysis indicated TCF4 as a novel target of miR-139-5p, our mechanistic studies validated this, and confirmed that TCF4 restoration could attenuate the tumor inhibitory activities of miR-139-5p in CC progression, and recover the normal Wnt/ $\beta$-catenin signaling. Conclusion: Our data collectively demonstrated that miR-139-5p was a vital tumor suppressor in $\mathrm{CC}$ pathogenesis via targeting TCF4 thereby inhibiting $\mathrm{Wnt} / \beta$-catenin signaling. The miR-139-5p/TCF4 axis may serve as a promising target for CC therapy.

Keywords: cervical cancer, miR-139-5p, TCF4, Wnt/ $\beta$-catenin signaling, tumor suppressor

\section{Introduction}

Cervical cancer (CC) now represents one of the most frequent gynecological malignancies in females all over the world, with increasing incidence and mortality every year especially in the developing countries, including in China. ${ }^{1-3}$ It is well accepted that persistent infection of high-risk human papillomavirus (HPV) is the main but not the only factor leading to the initial cervical carcinogenesis, ${ }^{4,5}$ whereas the precise mechanisms governing $\mathrm{CC}$ pathogenesis remain largely unclear. Current options for $\mathrm{CC}$ treatment include surgery, chemo- and radio-therapy. However, these strategies show finite interventions when $\mathrm{CC}$ patients are in the advanced stages, leaving their 
adverse 5-year survival rate at 30-50\%. ${ }^{6}$ Actually, metastasis and recurrence are the two most alarming causes leading to the poor outcomes of $\mathrm{CC}$ patients. ${ }^{7}$ Thus, keeping to explore more about the molecular mechanisms underlying is urgently needed to develop attractive targets for treating this disease.

MicroRNAs (miRNAs) are a group of short ( 22 nucleotides in length) RNA molecules with no proteincoding capacity. They are well known for their negative roles in regulating gene expression via post-transcription manners. ${ }^{8}$ Through base-pairing to the specific target sites among the $3^{\prime}$-untranslated region (3'-UTR) of certain genes, miRNAs effectively reduce the stability or inhibit the translation of these genes in either physiological or pathological conditions. ${ }^{9,10}$ Due to the wide distribution of these target genes, miRNAs serve as critical modulators in tumorigenesis by affecting key processes like cell proliferation, apoptosis, differentiation, angiogenesis, and metastasis. ${ }^{11,12}$ According to distinct cancer types or cellular context, the same miRNA might possess opposite biological functions, either as an oncogene or as a tumor suppressor. ${ }^{13}$ In cervical carcinogenesis, various miRNAs are aberrantly expressed and their dysregulation have been reported to have potential diagnostic and prognostic values in the clinic. ${ }^{14}$

MiR-139-5p, differing from miR-139-3p, is another mature miRNA form processed from the same miR-139 precursor. Although the tumor-suppressing roles of miR139-3p in CC have recently been demonstrated by scholars, ${ }^{15,16}$ the functions and mechanisms of miR-139$5 \mathrm{p}$ in CC remain unknown. Considering the common downregulation of miR-139-5p and tumor-suppressing effects in several other malignancies, ${ }^{17-19}$ we hypothesized that miR139-5p also exert tumor inhibitory activities in CC.

In this study, we started from the expression detection, and then conducted cellular assays and further mechanistic studies to characterize the biological roles and mechanisms of miR-139-5p in CC. Our convinced data illustrated that miR-139-5p could be developed as a novel diagnostic biomarker and therapeutic target for treating $\mathrm{CC}$.

\section{Materials and methods}

\section{Patients and tissues}

A total of 40 cases of cervical cancer tissues used in this study were collected at the Baotou Steel Hospital, Baotou, Inner Mongolia 014010, P.R. China between January 2015 and December 2018. Cervical cancer tissues and matched non-cancerous tissues were not received any chemotherapy or radiotherapy therapy before surgery. All tissue samples were frozen in liquid nitrogen immediately and stored at $-80^{\circ} \mathrm{C}$ for further analysis. All patients provided written informed consents in this study. This study was approved by the Ethical Committee of Baotou Steel Hospital, Baotou, Inner Mongolia 014010, P.R. China.

\section{Cell culture}

Four cervical cancer cell lines (CaSki, SiHa, Hela and C$33 \mathrm{~A}$ ) and one normal human cervix epithelial cell line (Ect1/E6E7) were purchased from American Type Culture Collection (ATCC, Manassas, VA, USA). Cells were cultured in Dulbecco's Modified Eagle's Medium (DMEM, Life Technology, Thermo Fisher Scientific, Waltham, MA, USA) containing $10 \%$ fetal bovine serum (FBS, Hyclone, Logan, UT, USA), $100 \mathrm{U} / \mathrm{mL}$ penicillin (Life Technology, Thermo Fisher Scientific, Waltham, MA, USA), and 100 $\mu \mathrm{g} / \mathrm{mL}$ streptomycin (Life Technology, Thermo Fisher Scientific, Waltham, MA, USA). Cell cultures were maintained in a humidified incubator at $37^{\circ} \mathrm{C}$ supplied with $5 \% \mathrm{CO}_{2}$.

\section{miRNA mimics and transfections}

MiR-139-5p mimics (Mimics) and microRNA negative controls (Con) were purchased by GenePharma Company (Shanghai, P.R. China). Transfections were performed by using Lipofectamine ${ }^{\mathrm{TM}} 2000$ Transfection Reagent (Life Technology, Thermo Fisher Scientific, Waltham, MA, USA) according to the manufacturer's instructions.

\section{qRT-PCR (quantitative real-time polymerase chain reaction)}

Total RNA was extracted from cells using TRIzol ${ }^{\circledR}$ reagent (Life Technology, Thermo Fisher Scientific, Waltham, MA, USA), according to the manufacturer's instructions. Total RNA was reverse transcribed into microRNA cDNAs with reverse Transcriptase M-MLV (RNase H-) (TaKaRa, Tokyo, Japan) by using specific primers (Ribobio, Guangzhou, P.R. China) according to the manufacturer's instructions. The cDNAs of gene were synthesized with M-MLV Reverse Transcriptase (Promega Corporation, Madison, WI, USA) according to the manufacturer's instructions. qRT-PCR was subsequently performed using the SYBR ${ }^{\circledR}$ Premix Ex Taq ${ }^{\mathrm{TM}}$ II kit (Takara Bio, Inc., Otsu, Japan). The relative expression levels were 
quantified using the $2^{-\Delta \Delta \mathrm{Ct}}$ method. U6 or GAPDH were used to do normalization as the internal reference gene.

\section{CCK-8 (cell counting kit-8) assay}

Twenty-four hours after transfection, cells were resuspended and seeded into 96-well plates at a density of $2 \times 10^{3}$ cells/well. Cell proliferation ability was detected by conducting a CCK-8 assay (Dojindo, Kumamoto, Japan) at three time points: 1, 2, and 3 Day after incubation. A total of $10 \mu \mathrm{L}$ CCK- 8 reagent was added to each well and incubated at $37^{\circ} \mathrm{C}$ with $5 \% \mathrm{CO}_{2}$ for another $2 \mathrm{hrs}$. Finally, the optical density of each well was measured at a wavelength of $450 \mathrm{~nm}$ using a microplate reader (Molecular Device, San Jose, CA, USA).

\section{EdU assays}

A 5-ethynyl-2'-deoxyuridine (EdU) incorporation assay was performed with a Cell-Light ${ }^{\mathrm{TM}}$ EdU Apollo ${ }^{\circledR} 567$ In Vitro Imaging kit (RiboBio, Guangzhou, China) according to the manufacturer's instructions. Twenty-four hours after transfection, cells were resuspended and seeded into 96well plates at a density of $2 \times 10^{3}$ cells/well. Cells were incubated with $50 \mu \mathrm{M}$ EdU for 2 hrs. The cells were then fixed in 4\% formaldehyde solution and stained with Apollo and Hoechst 33,342. Cells in four randomly selected microscopic fields were photographed and counted by a fluorescence microscope (Olympus Corporation, Tokyo, Japan).

\section{Transwell assay}

Twenty-four hours after transfection, SiHa or Hela cells were collected and suspended in serum-free medium. Subsequently, $3 \times 10^{5}$ cells were added to the upper chamber of 24-well Transwell chambers (Corning Incorporated, Corning, NY, USA) with a pore size of $8.0 \mu \mathrm{m}$. The lower chamber was filled with $500 \mu \mathrm{L}$ medium containing $10 \%$ FBS. Following incubation at $37^{\circ} \mathrm{C}$ for $24 \mathrm{hrs}$, cells below the membrane were fixed with $4 \%$ formaldehyde solution and stained with $0.25 \%$ crystal violet solution for $30 \mathrm{mins}$ at room temperature. After that, the cells were washed with PBS twice, air-dried and observed under an inverted microscope (Olympus Corporation, Tokyo, Japan). Number of migrated cells was counted in four randomly selected microscopic fields.

\section{Western blot analysis}

The protein was extracted by using radioimmunoprecipitation lysis buffer (RIPA buffer, Beyotime, Beijing, P.R. China) according to the manufacturer's instructions. An equal amount $(30 \mu \mathrm{g})$ samples were separated on $10 \%$ sodium dodecyl sulfatepolyacrylamide gel electrophoresis (SDS-PAGE) and transferred to nitrocellulose (NC) membrane (Millipore, Billerica, MA). The membrane was blocked by $5 \%$ skim milk for $1 \mathrm{hr}$ at room temperature. The membranes were incubated with the specific antibodies with TCF4, GAPDH, c-myc, cyclin D1, MMP9, and $\beta$-catenin (Santa Cruz Biotechnology, Santa Cruz, CA, USA) overnight at $4^{\circ} \mathrm{C}$. After incubation with secondary antibodies (Beyotime, Beijing, P.R. China) for $1 \mathrm{hr}$ at room temperature, the blots were detected using an enhanced chemiluminescence detection system (Pierce, Billerica, MA). GAPDH was used as an internal control.

\section{Plasmid construction}

The wild type fragment of TCF4 3'-UTR (WT) containing the miR-139-5p predicted binding site or mutant fragment of TCF4 3'-UTR (MT) was synthesized by BGI Tech (Beijing, P.R. China). The two fragments were sub-cloned into pmirGLO vector (Promega Corporation, Madison, WI, USA). The coding sequence of TCF4 mRNA was amplified by PCR using KOD-plus-Ver.2 kit (KOD-211, TOYOBO, Osaka, Japan) and subcloned into pcDNA3.1 expression vector.

\section{Luciferase reporter assay}

For TOP Flash assay: SiHa or Hela cells were seeded in 24 well plates and co-transfected with TOP Flash firefly luciferase plasmid, renilla luciferase plasmid, and miR-139-5p mimics (Mimics) or controls (Con). 48 hrs later, luciferase activity was determined using the Dual-Luciferase ${ }^{\circledR}$ Reporter Assay System (Promega Corporation, Madison, WI, USA) according to the manufacturer's instructions, and relative $\mathrm{Wnt} / \beta$-catenin activity was calculated. For TCF4 3'-UTR assay: SiHa or Hela cells were co-transfected with miR-139-5p mimics (Mimics) or controls (Con) and luciferase reporter vectors containing the wild type (WT) of mutant (MT) TCF4 3'-UTR sequences of the miR-139-5p binding sites. 48 hrs later, luciferase activity was detected using Dual-Luciferase ${ }^{\circledR}$ Reporter Assay System (Promega Corporation, Madison, WI, USA) according to the manufacturer's instructions.

\section{Target prediction}

Targets of miR-139-5p were searched on Targetscan Release 7.2 (http://www.targetscan.org/vert_72/), and the results suggesting that TCF4 is a potential target of miR-139-5p. To further confirm that TCF4 is a direct target of miR-139-5p, 
we obtained more information about the $3^{\prime}$-UTR of TCF4 mRNAs on Targetscan Release 7.2.

\section{Statistical analysis}

Statistical analyses were performed by GraphPad Prism 6 software (GraphPad Software, Inc., La Jolla, USA). Statistical significance of difference between groups was determined by a two-tailed paired or grouped Student's $t$ test. The correlation between miR-139-5p and TCF4 expressions was analyzed by Spearman's correlation analysis. Data were expressed as mean $\pm \mathrm{SD}$ from at least three independent experiments. A $P$-value of less than 0.05 was considered to indicate statistical significance.

\section{Results}

\section{Decreased expression of miR-139-5p in $\mathrm{CC}$ and its association with lymph node metastasis of CC patients}

To explore the exact role of miR-139-5p in CC, we firstly analyzed its expression status in collected cervical cancer tissues paired with adjacent non-cancerous tissues. qRTPCR results clearly showed that miR-139-5p expression was frequently decreased in these 40 cases of CC tissues when compared with the matched non-cancerous tissues, and the difference was of significance (Figure 1A, $P<0.05)$. Notably, miR-139-5p was downregulated in CC tissues with lymph node metastasis than those without (Figure 1B, $P<0.05$ ). Namely, the lower miR-139-5p was expressed, the more positive metastasis was observed in $\mathrm{CC}$ patients. Meanwhile, we also determined miR-139-5p expression in four CC cell lines (CaSki, SiHa, Hela, and C-33A) and one normal cervix epithelial cell line (Ect1/ E6E7). MiR-139-5p was also downregulated in these CC cell lines compared to the normal one (Figure 1C). Hence, the expression status detection verified the common decrease of miR-139-5p level in CC tissues and cell lines. These findings collectively suggested that decreased miR-139-5p expression might be tightly connected with $\mathrm{CC}$ progression.

\section{Mir-139-5p inhibits CC cell proliferation and migration in vitro and blocks the Wnt/ $\beta$-catenin signaling}

Since SiHa and Hela, the two CC cell lines contained relatively lower levels of miR-139-5p (Figure 1C), they were further used in the subsequent cellular experiments. Gain-offunction assays were performed by transfecting $\mathrm{SiHa}$ and Hela cells with miR-139-5p mimics (Mimics). qRT-PCR validated the effective overexpression of miR-139-5p in the two cell lines (Figure 2A). Then, cell proliferation was monitored by CCK- 8 and EdU assays. The results showed that $\mathrm{SiHa}$ and Hela cells with miR-139-5p overexpression (by transfecting miR-139-5p mimics) exhibited markedly growth suppression than those transfected with microRNA negative controls (Con) (Figure 2B and C). These results indicated that miR-139-5p itself was a strong inhibitor on CC cell proliferation. Considering the above correlation of lower miR-139-5p expression with positive lymph node metastasis, we suspected that it also impacted on CC cell migration. Transwell assay revealed that $\mathrm{SiHa}$ and Hela cells with miR-139-5p overexpression showed obvious migration inhibition than the control groups (Figure 2D). Therefore, our in vitro experiments demonstrated that ectopic miR-139-5p expression inhibited CC cell proliferation and migration. In other words, miR-139-5p exerts tumor-suppressing roles in CC progression.
A

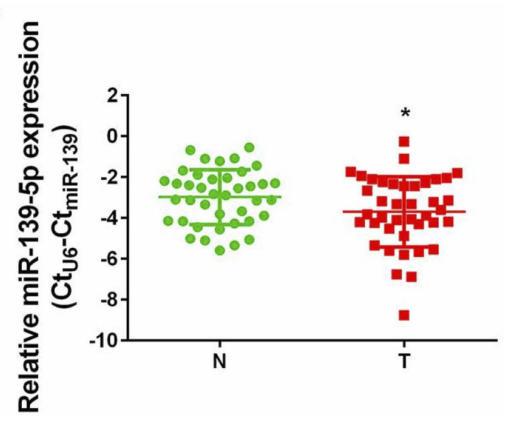

B

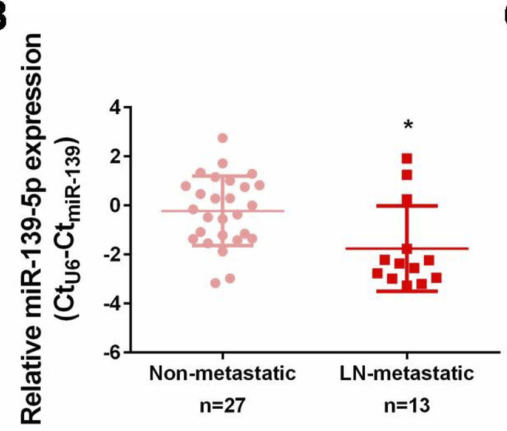

C

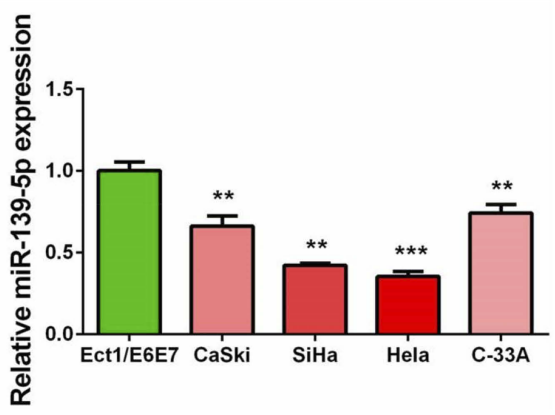

Figure I Decreased expression of miR-139-5p in cervical cancer tissues and cell lines. (A) qRT-PCR analysis was performed to detect miR-139-5p expression in 40 pairs of cervical cancer tissues and matched non-cancerous tissues. N: normal, T: tumor. (B) qRT-PCR analysis was performed to detect miR-I39-5p expression in lymph node metastatic CC tissues $(n=13)$ compared with non-metastatic CC tissues $(n=27)$. LN: lymph node. (C) qRT-PCR analysis was performed to detect miR-I39-5p expression in four cervical cancer cell lines (CaSki, SiHa, Hela, and C-33A) and one normal human cervix epithelial cell line $(\mathrm{Ect} \mathrm{I} / \mathrm{E} 6 \mathrm{E} 7) . * P<0.05, * * P<0.0 \mathrm{I}, * * * P<0.00 \mathrm{I}$. 
A

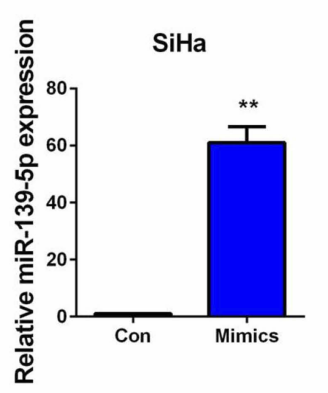

C

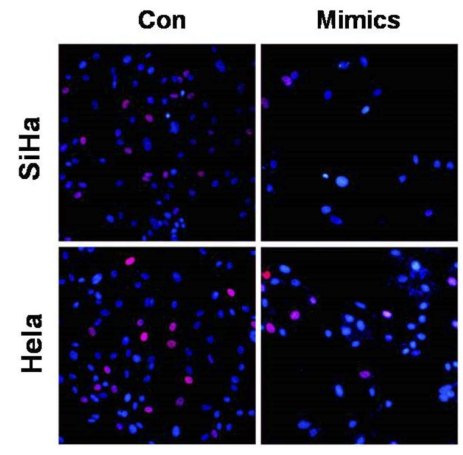

B
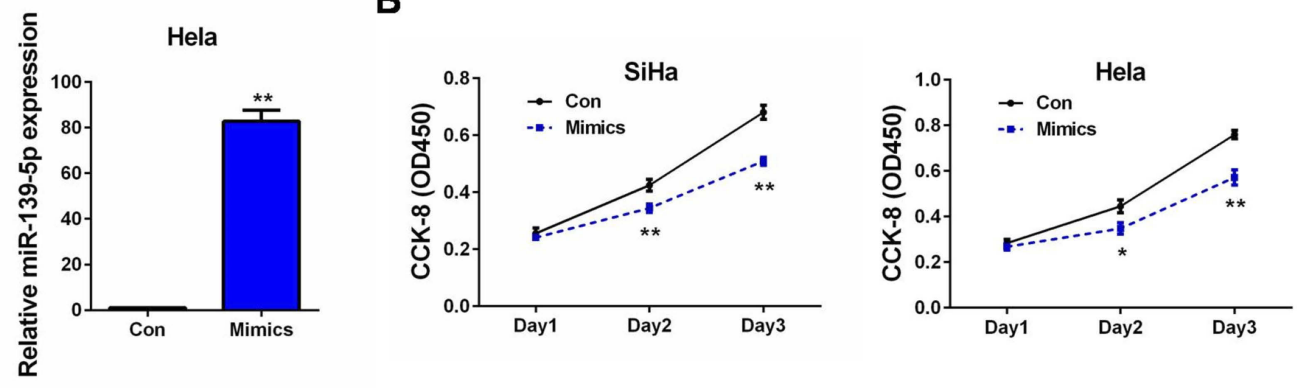

D

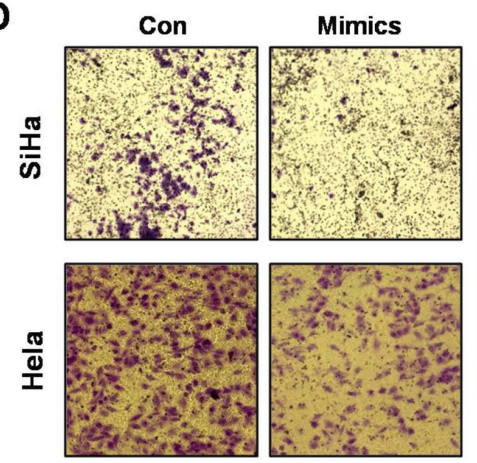

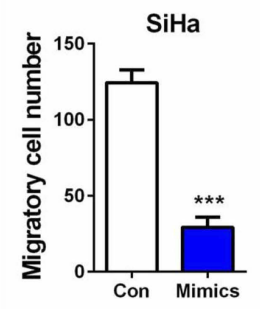

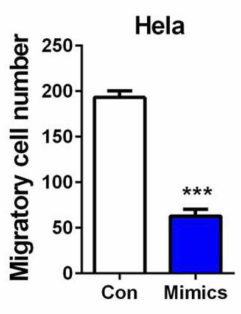

Figure 2 miR-139-5p inhibits CC cell proliferation and migration in vitro. (A) SiHa or Hela cells were transfected with miR-139-5p mimics (Mimics) or controls (Con). 24 hrs later, relative miR-139-5p expression was examined by qRT-PCR, respectively. (B) SiHa or Hela cells were transfected with miR-139-5p mimics (Mimics) or controls (Con). At 24, 48, and 72 hrs after transfection, CCK-8 assay was performed to detect the cell proliferation ability, respectively. (C) SiHa or Hela cells were transfected with miR-139-5p mimics (Mimics) or controls (Con). 24 hrs later, EdU assay was performed to detect the cell proliferation ability, respectively. (D) SiHa or Hela cells were transfected with miR-139-5p mimics (Mimics) or controls (Con). 24 hrs later, transwell assay was performed to detect the cell migration ability, respectively. Scale bar: 200

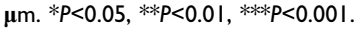

Since activated Wnt/ $\beta$-catenin signaling is closely related to tumorigenesis of multiple cancers, including $\mathrm{CC},{ }^{20}$ we then measured the luciferase activities of the TOP Flash reporter in miR-139-5p overexpressed CC cells. Surprisingly, the TOP Flash reporter activities were significantly inhibited by miR-139-5p overexpression in the two CC cell lines (Figure 3A). Moreover, this pathway inhibition was accompanied by decrease of c-myc, cyclin D1, and MMP9 mRNAs and proteins, whereas minor alternation on $\beta$-catenin expression (Figure $3 \mathrm{~B}$ and $\mathrm{C}$ ). These results indicated that the tumor-suppressing roles of miR-139-5p were to some extent associated with its blocking effect on the $\mathrm{Wnt} / \beta$-catenin signaling, despite in a $\beta$-catenin-independent manner.

\section{TCF4 is a direct target of miR-139-5p in $\mathrm{CC}$}

To further explore the molecular mechanism of miR-139-5p in CC, especially to understand its connection with the Wnt/ $\beta$-catenin signaling, we then conducted bioinformatics analysis by utilizing Targetscan Release 7.2 (http://www.targets can.org/vert_72/) to search the putative targets of miR-139$5 p$. Interestingly, we observed a highly conserved potential binding site in the 3'-UTR of transcription factor 4 (TCF4) mRNA (Figure 4A). As is known, TCF4 is a crucial transcription factor collaborating with $\beta$-catenin to initiate the transcription of target genes in response to Wnt activation. ${ }^{21}$ We then selected TCF4 for further investigation according to the above tumor-suppressing roles of miR-139-5p and its negative impact on the $\mathrm{Wnt} / \beta$-catenin signaling. A wild type TCF4-3'-UTR luciferase reporter (WT) containing the putative binding site of miR-139-5p was first constructed. We found that miR-139-5p overexpression led to a marked reduction of the luciferase activity of this wild type reporter (Figure 4B). However, when the binding site of miR-139-5p was deleted in this reporter (MT), miR-139-5p overexpression failed to exert the inhibitory effect in the two CC cell lines (Figure 4B). In addition, our qRT-PCR and Western blot assays also verified that TCF4 expression was suppressed in the miR-139-5p-overexpressing cells (Figure 4C and D). Therefore, the target verification experiments confirmed TCF4 as a novel target of miR-139-5p in CC cells. 
A
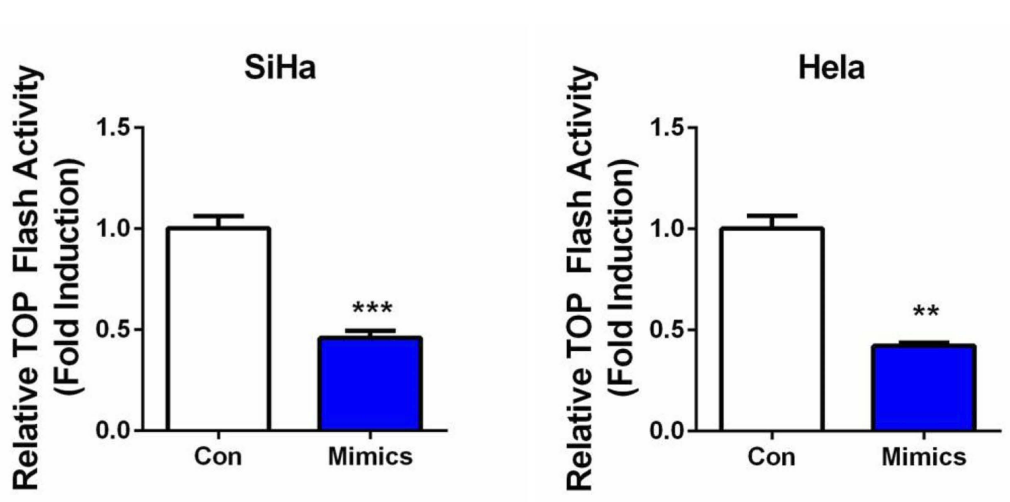

C

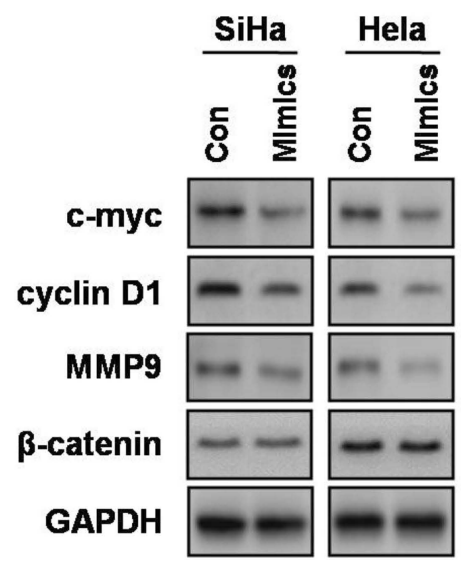

B
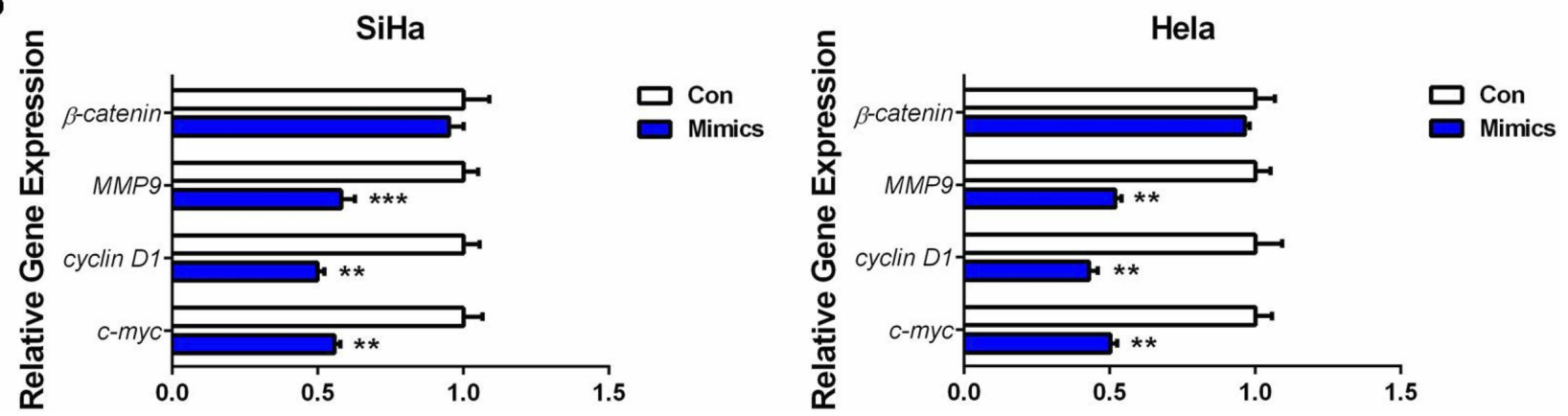

Figure 3 MiR-139-5p inhibits Wnt/ק-catenin signaling in CC. (A) SiHa or Hela cells were co-transfected with TOP Flash firefly luciferase plasmid, renilla luciferase plasmid, and miR-139-5p mimics (Mimics) or controls (Con). 48 hrs later, luciferase activity was determined, and relative Wnt/B-catenin activity was calculated. (B) SiHa or Hela cells were transfected with miR-139-5p mimics (Mimics) or controls (Con). 24 hrs later, qRT-PCR was performed to detect the mRNA expressions of Wnt target genes, as well as $\boldsymbol{\beta}$-catenin. (C) $\mathrm{SiH}$ or Hela cells were transfected with miR-139-5p mimics (Mimics) or controls (Con). 24 hrs later, Western blot analysis was performed to detect the protein expressions of $\mathrm{Wnt}$ target genes, as well as $\boldsymbol{\beta}$-catenin. $* * P<0.01$, $* * * P<0.001$.

\section{Restoration of TCF4 attenuates the suppressing roles of miR-139-5p in CC progression and recovers the $\mathrm{Wnt} / \beta$-catenin signaling}

To affirm whether miR-139-5p inhibited CC cell proliferation and migration via targeting TCF4, we performed rescue experiments. Briefly, TCF4 expression plasmid was transfected as a control group or co-transfected with miR-139-5p mimics to establish the restoration group in $\mathrm{SiHa}$ cells (Figure 5A and B). Quite in agreement with our hypothesis, TCF4 could significantly promote cell proliferation and migration, while restoration of TCF4 in miR-139-5p-overexpressing cells resulted in cell proliferation and migration recovery to the levels of the control group (Con, co-transfected with the blank vector plasmid and microRNA negative controls; Figure $5 \mathrm{C}$ and D). Detections of the Wnt $/ \beta$-catenin signaling, including the luciferase activity of the TOP Flash reporter, the mRNA levels, and the protein expressions of cmyc, cyclin D1, and MMP9, further verified that TCF4 restoration recovered the activities of the $\mathrm{Wnt} / \beta$-catenin signaling (Figure $5 \mathrm{E}-\mathrm{G}$ ). These results clearly demonstrated that miR-139-5p inhibits CC progression at least partially via downregulating TCF4.

\section{Increased expression of TCF4 and its inverse correlation with miR-139-5p expression in CC}

From the above findings, we have already identified the effects of miR-139-5p/TCF4 axis in regulating Wnt/ $\beta$-catenin signaling and $\mathrm{CC}$ progression. We then examined the expression of TCF4 in CC tissues to further evaluate the relationship between miR-139-5p and TCF4. In the aforementioned 40 pairs of $\mathrm{CC}$ tissues, we found that TCF4 mRNA was higher expressed in cervical cancer tissues than that in the matched non-cancerous tissues (Figure 6A, $P<0.05)$. Consistently, TCF4 was upregulated in CC tissues with lymph node metastasis than those without (Figure 6B, $P<0.05)$. The immunohistochemistry analyses from the 
A

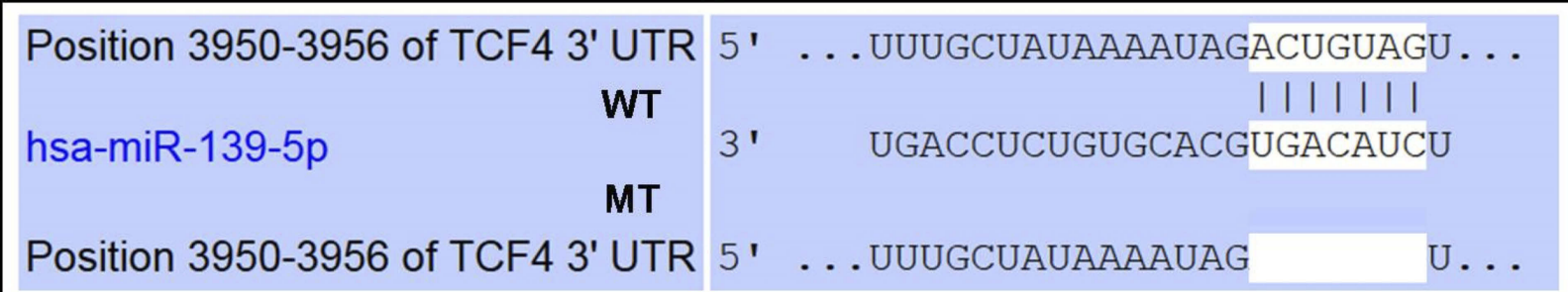

B
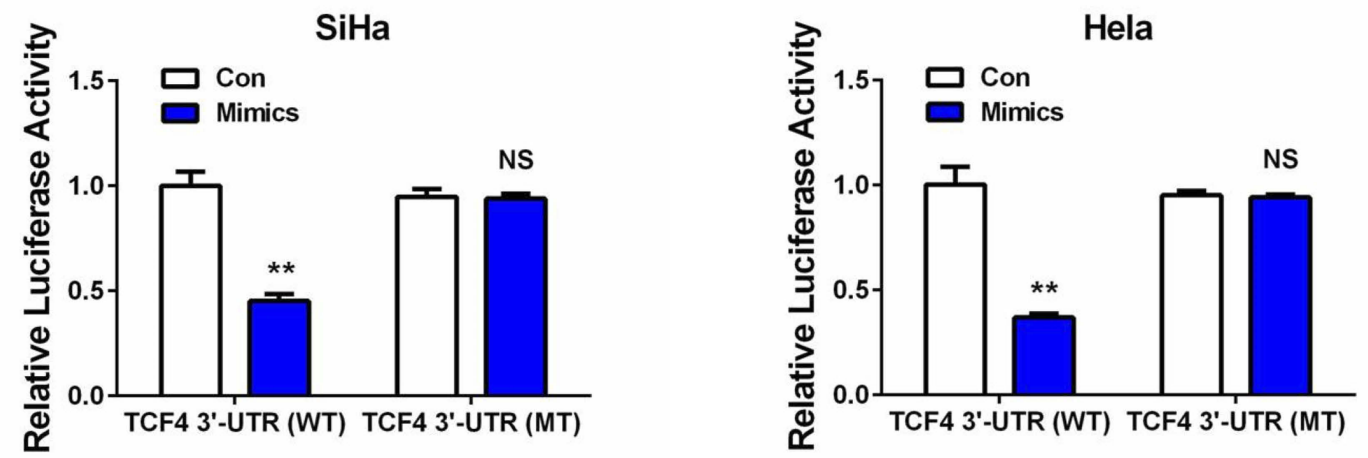

C
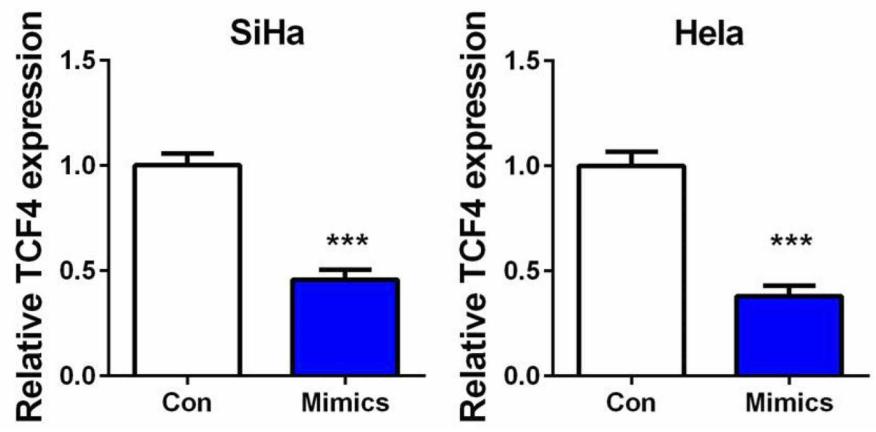

D

\begin{abstract}
Figure 4 TCF4 is a direct target of miR-139-5p in CC. (A) Sequence of the miR-139-5p binding site with wild type (WT) TCF4 3'-UTR (top) and the mutant (MT) TCF4 3'UTR sequence (bottom). (B) SiHa or Hela cells were co-transfected with miR-139-5p mimics (Mimics) or controls (Con) and luciferase reporter vectors containing the wild type of mutant TCF4 3'-UTR sequences of the miR-139-5p binding sites. 48 hrs after transfection, luciferase activities were measured. (C) qRT-PCR analysis of TCF4 mRNA expression after SiHa or Hela cells was transfected with miR-139-5p mimics (Mimics) or controls (Con). (D) Western blot analysis of TCF4 protein expression after SiHa or Hela cells was transfected with miR-139-5p mimics (Mimics) or controls (Con). **P<0.0I, ***P<0.001.

Abbreviation: NS, no significance.
\end{abstract}

human protein atlas (https://www.proteinatlas.org/) validated its protein upregulation in $\mathrm{CC}$ tissues (Figure 6C). Moreover, an inverse correlation was found between miR-139-5p and TCF4 mRNA levels in the 40 cases of paired CC tissues (Figure 6D). Collectively, we validated a negative correlation of the two molecules, and confirmed the above targeting relation (miR-139-5p directly targets TCF4) in the expression aspect. Our findings fully support that TCF4 is critical factor in guiding CC progression.

\section{Discussion}

Cervical cancer (CC) is the most malignant cancer affecting women, with still high morbidity and mortality in the developing countries. Statistically, nearly a quarter of all CC patients are in China. ${ }^{2,3}$ Recently, although some progress has been achieved in the diagnostic veracity of cervical lesions, the clinical outcome remains unsatisfactory. ${ }^{6}$ MiRNAs have been found to be dysregulated in the early stages of $\mathrm{CC}$ and they contribute to cervical carcinogenesis by directly downregulating tumor-associated genes thus impacting on multiple malignant behaviors. ${ }^{22,23}$ Studies focusing on the specific roles and mechanisms of miRNAs in CC may aid in the efficiency in diagnosis, treatment, and prognosis of this disease. In this study, we analyzed the expression, biological function, and possible mechanisms of miR-139-5p, a 
A

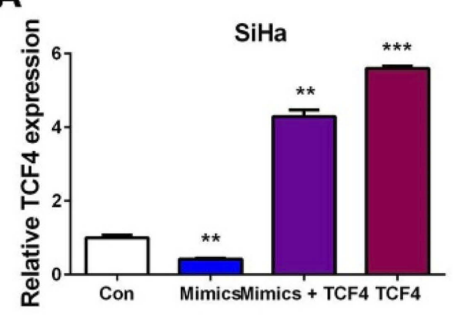

B

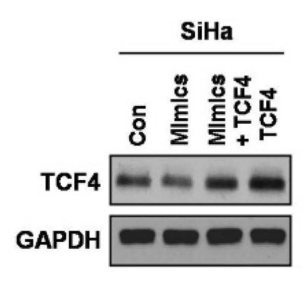

C

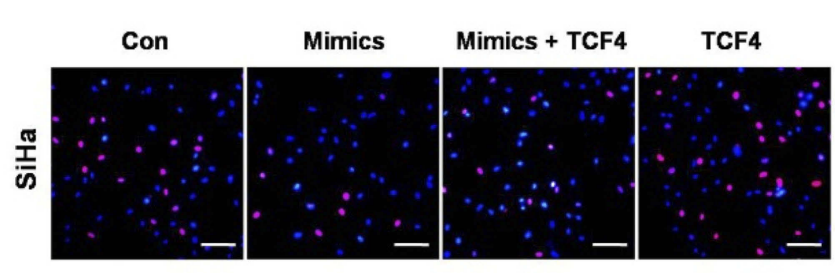

D
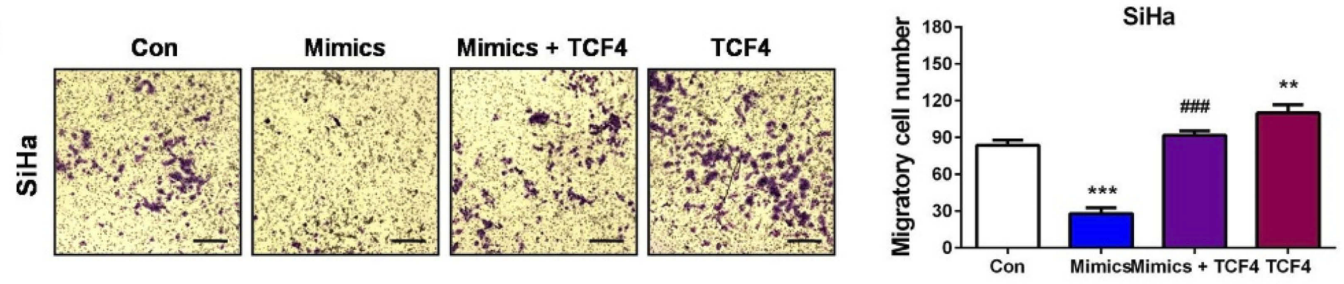

E

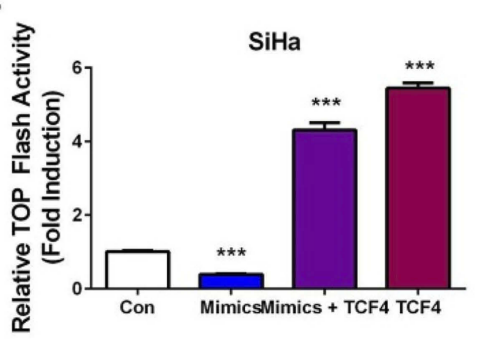

F

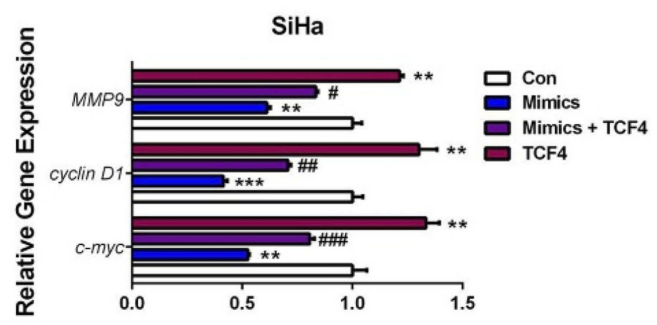

G

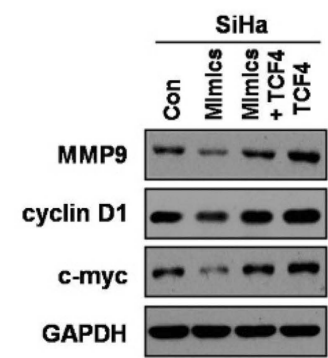

Figure 5 Restoration of TCF4 attenuates the suppressive roles of miR-139-5p in CC progression and recovers the $\mathrm{Wnt} / \boldsymbol{\beta}$-catenin signaling activity. (A) SiHa cells were transfected with miR-139-5p mimics (Mimics), miR-139-5p mimics plus TCF4 expression plasmids (Mimics+TCF4), TCF4 expression plasmids (TCF4) or controls (Con). 24 hrs later, qRT-PCR was performed to detect the mRNA expression of TCF4. (B) SiHa cells were transfected with miR-139-5p mimics (Mimics), miR-139-5p mimics plus TCF4 expression plasmids (Mimics+TCF4), TCF4 expression plasmids (TCF4) or controls (Con). 24 hrs later, Western blot analysis was performed to detect the protein expression of TCF4. (C) SiHa cells were transfected with miR-139-5p mimics (Mimics), miR-139-5p mimics plus TCF4 expression plasmids (Mimics+TCF4), TCF4 expression plasmids (TCF4) or controls (Con). 24 hrs later, EdU assay was performed to detect the cell proliferation ability. (D) SiHa cells were transfected with miR139-5p mimics (Mimics), miR-139-5p mimics plus TCF4 expression plasmids (Mimics+TCF4), TCF4 expression plasmids (TCF4) or controls (Con). 24 hrs later, transwell assay was performed to detect the cell migration ability. (E) SiHa cells were co-transfected with TOP Flash firefly luciferase plasmid, renilla luciferase plasmid, miR-I39-5p mimics (Mimics) or miR-139-5p mimics plus TCF4 expression plasmids (Mimics+TCF4), TCF4 expression plasmids (TCF4) or controls (Con). 48 hrs later, luciferase activity was determined, and relative Wnt/ $\boldsymbol{\beta}$-catenin activity was calculated. (F) SiHa cells were transfected with miR-139-5p mimics (Mimics), miR-139-5p mimics plus TCF4 expression plasmids (Mimics+TCF4), TCF4 expression plasmids (TCF4) or controls (Con). 24 hrs later, qRT-PCR was performed to detect the mRNA expressions of Wnt target genes. (G) SiHa cells were transfected with miR-139-5p mimics (Mimics), miR-139-5p mimics plus TCF4 expression plasmids (Mimics+TCF4), TCF4 expression plasmids (TCF4) or controls (Con). 24 hrs later, Western blot analysis was performed to detect the protein expressions of Wnt target genes. Scale bar: $200 \mu \mathrm{m}$. $* * P<0.0 \mathrm{I}$, $* * * P<0.001$, compared with Con group. \#P<0.05, \#P<0.01, \#\#P<0.001, compared with Mimics group.

poorly known tumor suppressor, in CC progression. Our results supported that decreased miR-139-5p could be used as a therapeutic target of $\mathrm{CC}$.

Except miR-139-3p, miR-139-5p is another mature miRNA product from the same miR-139 precursor. Through reviewing literatures, we noticed that two groups had illustrated the tumor-suppressing roles of miR-139-3p in CC. ${ }^{15,16}$ They proved that miR-139-3p, downregulated by host gene DNA methylation mediated by HPV-16, could inhibit cervical oncogenesis by suppressing cell proliferation, migration, invasion and inducing apoptosis. ${ }^{15,16}$ However, no report has characterized the role of miR-139$5 p$ in CC. We designed a series of experiments, first found that miR-139-5p was decreased in CC tissues, especially in those with lymph node metastasis. Its downregulation was also confirmed in several CC cell lines. The decrease of miR-139-5p in CC met expectations, since it was processed from the same miR-139 precursor with miR-139-3p. Much less, mountains of evidences have reported the downregulation of miR-139-5p in other types of malignancies. ${ }^{17-19}$ Our expression detection, on the one hand, uncovered that miR139-5p was decreased in CC, on the other hand suggested its connection with $\mathrm{CC}$ progression.

To recognize the biological roles of miR-139-5p in CC, we performed gain-of-function assays in the two CC lines (SiHa and Hela) harboring lower endogenous miR-139-5p levels. Transfecting them with miR-139-5p mimics successfully overexpressed this miRNA instantaneously. Functional assays including CCK-8, EdU, and transwell assays demonstrated that miR-139-5p strongly inhibited 


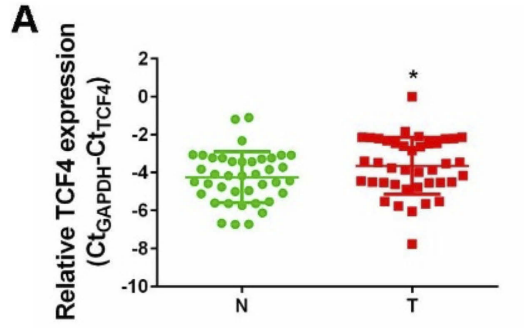

B

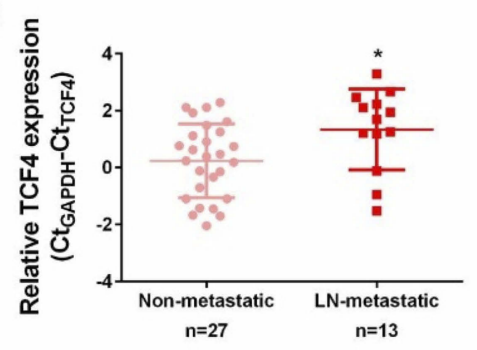

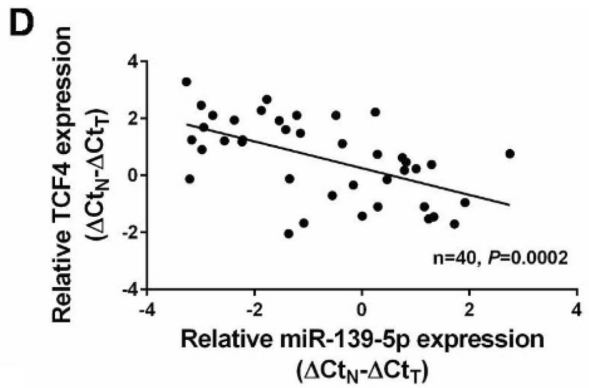
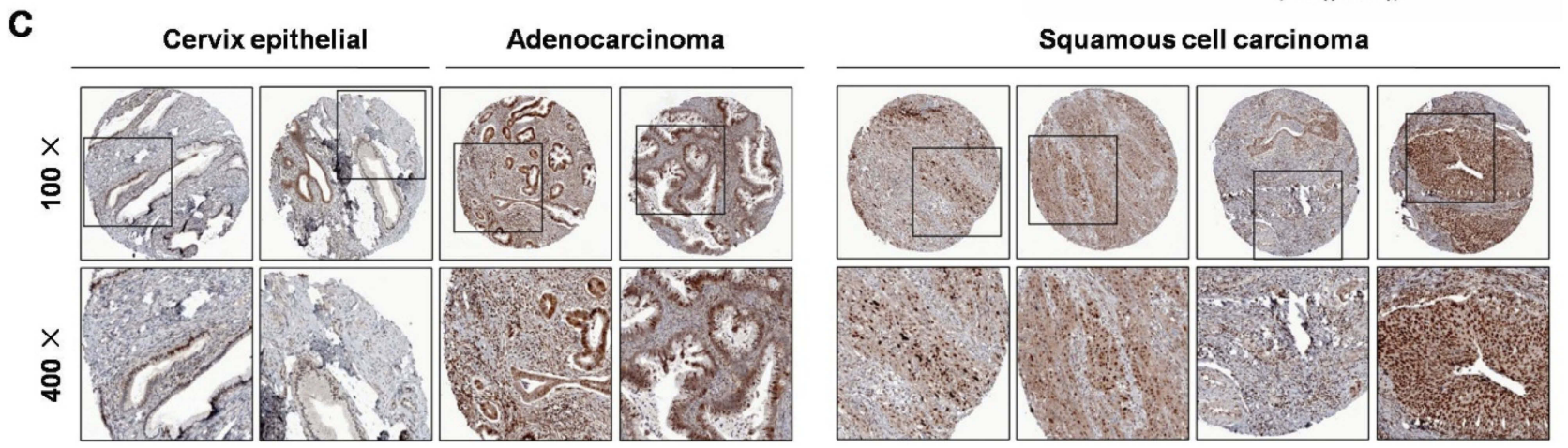

Figure 6 Increased expression of TCF4 and its inverse correlation with miR-139-5p expression in CC. (A) qRT-PCR analysis was performed to detect TCF4 expression in 40 pairs of cervical cancer tissues and matched non-cancerous tissues. N: normal, T: tumor. (B) qRT-PCR analysis was performed to detect TCF4 expression in lymph node metastatic CC tissues $(n=13)$ compared with non-metastatic CC tissues $(n=27)$. LN: lymph node. $(\mathbf{C})$ Immunohistochemistry analyses from the online tool human protein atlas (https://www.proteinatlas.org/) validated TCF4 protein upregulation in CC tissues. Up panel: I00x, down panel: 200x. (D) Spearman's correlation analysis was performed to explore the negative correlation between miR-139-5p and TCF4 mRNA levels in paired CC tissues $(n=40, P=0.0002)$. $* P<0.05$.

$\mathrm{CC}$ cell proliferation and migration in vitro. Hence, together with the above downregulation of miR-139-5p in $\mathrm{CC}$, we verified that this miRNA could be developed as a valuable therapeutic agent for $\mathrm{CC}$ patients.

Activation of the $\mathrm{Wnt} / \beta$-catenin signaling is tightly connected with cervical carcinogenesis, ${ }^{20}$ and several key components of this signaling pathway have been identified as oncogenic factors in CC. ${ }^{24-26}$ Surprisingly, our cellular experiments found that miR-139-5p overexpression led to the Wnt/ $\beta$-catenin signaling blockage, as detected by the TOP Flash reporter and downstream target gene expression of this pathway. Therefore, we suspected that the tumor-suppressing roles of miR-139-5p were to some extent associated with its inhibitory effect on the Wnt/ $\beta$ catenin signaling.

During the screen and validation of the direct targets of miR-139-5p, we observed that TCF4, the important transcription factor collaborating with $\beta$-catenin to initiate the transcription of target genes in response of Wnt activation, ${ }^{21}$ was a putative target of miR-139-5p, since it contained a highly conserved miR-139-5p binding site in the 3'-UTR. We then focused on TCF4 and conducted several experiments to confirm whether its targeting by miR-139-5p truly existed. The results came out that TCF4 was a real and direct target of miR-139-5p. Moreover, subsequent TCF4 restoration in miR-139-5p-overexpressing cells further supported that miR-139-5p inhibited CC progression at least partially via downregulating TCF4, since TCF4 restoration not only attenuated the suppressing roles of miR-139-5p in CC progression, but also recovered the Wnt/ $\beta$-catenin signaling. Finally, qRT-PCR and IHC confirmed the upregulation of TCF4 in CC tissues, inversely correlated with the downregulation of miR-139-5p. These results once more verified that there was a targeting relationship between TCF4 and miR-139-5p.

Taken together, our findings identified miR-139-5p as an important tumor suppressor in CC by inhibiting cell proliferation and migration. The direct targeting of TCF4, the crucial transcription factor of the Wnt/ $\beta$-catenin signaling by miR139-5p finely explained its blocking effect on this signaling pathway and tumor inhibitory activities in CC. However, the valid in vivo evidences are still required to prove the tumorsuppressing roles of miR-139-5p in CC. Nevertheless, our data provide a strong foundation for the future application of miR-139-5p as a novel target for CC therapy.

\section{Disclosure}

The authors report no conflicts of interest in this work. 


\section{References}

1. Siegel RL, Miller KD, Jemal A. Cancer statistics, 2016. CA Cancer J Clin. 2016;66(1):7-30. doi:10.3322/caac.21332

2. Chen W, Zheng R, Baade PD, et al. Cancer statistics in China, 2015. CA Cancer J Clin. 2016;66(2):115-132. doi:10.3322/caac.21338

3. Di J, Rutherford S, Chu C. Review of the cervical cancer burden and population-based cervical cancer screening in China. Asian Pac J Cancer Prev. 2015;16(17):7401-7407. doi:10.7314/apjcp.2015.16.17.7401

4. Banister CE, Liu C, Pirisi L, Creek KE, Buckhaults PJ. Identification and characterization of HPV-independent cervical cancers. Oncotarget. 2017;8(8):13375-13386. doi:10.18632/oncotarget.14533

5. He C, Lv X, Huang C, et al. A human papillomavirus-independent cervical cancer animal model reveals unconventional mechanisms of cervical carcinogenesis. Cell Rep. 2019;26(10):2636-2650.e5. doi:10. 1016/j.celrep.2019.02.004

6. Wright JD, Huang Y, Ananth CV, et al. Influence of treatment center and hospital volume on survival for locally advanced cervical cancer. Gynecol Oncol. 2015;139(3):506-512. doi:10.1016/j.ygyno.2015.07.015

7. Lokich E. Gynecologic cancer survivorship. Obstet Gynecol Clin North Am. 2019;46(1):165-178. doi:10.1016/j.ogc.2018.10.002

8. Bartel DP. MicroRNAs: target recognition and regulatory functions. Cell. 2009;136(2):215-233. doi:10.1016/j.cell.2009.01.002

9. Lorente-Cebrián S, González-Muniesa P, Milagro FI, Martínez JA. MicroRNAs and other non-coding RNAs in adipose tissue and obesity: emerging roles as biomarkers and therapeutic targets. Clin Sci (Lond). 2019;133(1):23-40. doi:10.1042/CS20180890

10. Lu J, Getz G, EA M, et al. MicroRNA expression profiles classify human cancers. Nature. 2005;435(7043):834-838. doi:10.1038/ nature 03702

11. Calin GA, Croce CM. MicroRNA signatures in human cancers. Nat Rev Cancer. 2006;6(11):857-866. doi:10.1038/nrc1997

12. Ors-Kumoglu G, Gulce-Iz S, Biray-Avci C. Therapeutic microRNAs in human cancer. Cytotechnology. 2019;71(1):411-425. doi:10.1007/ s10616-018-0291-8

13. Yu T, Ma P, Wu D, Shu Y, Gao W. Functions and mechanisms of microRNA-31 in human cancers. Biomed Pharmacother. 2018;108:1162-1169. doi:10.1016/j.biopha.2018.09.132

14. Li J, Liu Q, Clark LH, Qiu H, Bae-Jump VL, Zhou C. Deregulated miRNAs in human cervical cancer: functional importance and potential clinical use. Future Oncol. 2017;13(8):743-753. doi:10.2217/fon2016-0328
15. Sannigrahi MK, Sharma R, Singh V, Panda NK, Rattan V, Khullar M. Role of host miRNA Hsa-miR-139-3p in HPV-16-induced carcinomas. Clin Cancer Res. 2017;23(14):3884-3895. doi:10.1158/10780432.CCR-16-2936

16. Huang P, Xi J, Liu S. MiR-139-3p induces cell apoptosis and inhibits metastasis of cervical cancer by targeting NOB1. Biomed Pharmacother. 2016;83:850-856. doi:10.1016/j.biopha.2016.07.050

17. Li P, Xiao Z, Luo J, Zhang Y, Lin L. MiR-139-5p, miR-940 and miR$193 a-5 p$ inhibit the growth of hepatocellular carcinoma by targeting SPOCK1. J Cell Mol Med. 2019;23(4):2475-2488. doi:10.1111/ jcmm. 14121

18. Yang B, Zhang W, Sun D, et al. Downregulation of miR-139-5p promotes prostate cancer progression through regulation of SOX5. Biomed Pharmacother. 2019;109:2128-2135. doi:10.1016/j.biopha. 2018.09.029

19. Jiao W, Zhang J, Wei Y, et al. MiR-139-5p regulates VEGFR and downstream signaling pathways to inhibit the development of esophageal cancer. Dig Liver Dis. 2019;51(1):149-156. doi:10.1016/j. dld.2018.07.017

20. Anastas JN, Moon RT. WNT signalling pathways as therapeutic targets in cancer. Nat Rev Cancer. 2013;13(1):11-26. doi:10.1038/ $\operatorname{nrc} 3419$

21. Sareddy GR, Panigrahi M, Challa S, Mahadevan A, Babu PP. Activation of Wnt/beta-catenin/Tcf signaling pathway in human astrocytomas. Neurochem Int. 2009;55(5):307-317. doi:10.1016/j. neuint.2009.03.016

22. Wang $\mathrm{F}, \mathrm{Li} \mathrm{B}$, Xie $\mathrm{X}$. The roles and clinical significance of microRNAs in cervical cancer. Histol Histopathol. 2016;31(2):131139. doi:10.14670/HH-11-666

23. Srivastava SK, Ahmad A, Zubair H, et al. MicroRNAs in gynecological cancers: small molecules with big implications. Cancer Lett. 2017;28(407):123-138. doi:10.1016/j.canlet.2017.05.011

24. Liu XF, Li XY, Zheng PS, Yang WT. DAX1 promotes cervical cancer cell growth and tumorigenicity through activation of $\mathrm{Wnt} / \beta$-catenin pathway via GSK3 $\beta$. Cell Death Dis. 2018;9(3):339. doi:10.1038/ s41419-018-1111-y

25. Sun X, Liu Y. Activation of the Wnt/ $\beta$-catenin signaling pathway may contribute to cervical cancer pathogenesis via upregulation of twist. Oncol Lett. 2017;14(4):4841-4844. doi:10.3892/ol.2017.6754

26. Bahrami A, Hasanzadeh M, ShahidSales S, et al. Clinical significance and prognosis value of Wnt signaling pathway in cervical cancer. $J$ Cell Biochem. 2017;118(10):3028-3033. doi:10.1002/jcb.25992
OncoTargets and Therapy

\section{Publish your work in this journal}

OncoTargets and Therapy is an international, peer-reviewed, open access journal focusing on the pathological basis of all cancers, potential targets for therapy and treatment protocols employed to improve the management of cancer patients. The journal also focuses on the impact of management programs and new therapeutic agents and protocols on patient perspectives such as quality of life, adherence and satisfaction. The manuscript management system is completely online and includes a very quick and fair peer-review system, which is all easy to use. Visit http://www.dovepress.com/ testimonials.php to read real quotes from published authors. 